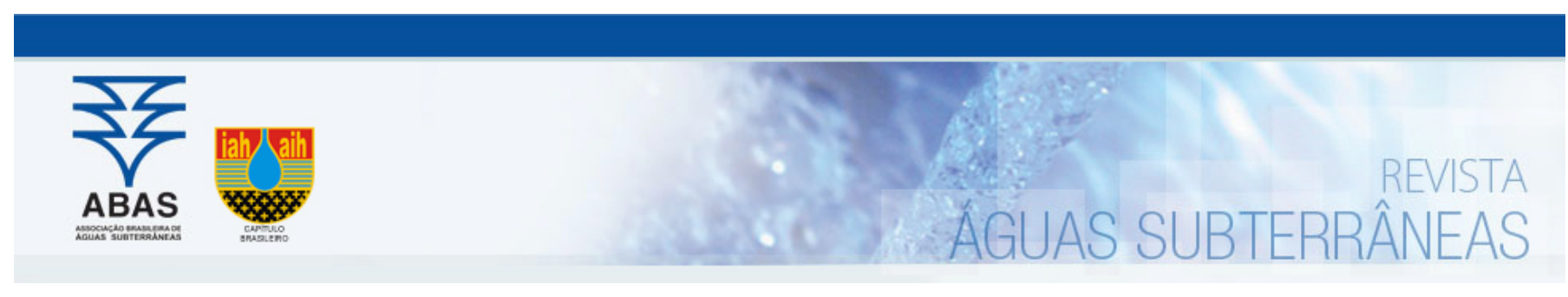

Artigos

\title{
Modelo Hidrogeológico Conceitual da Estação Ecológica de Santa Bárbara (EEcSB) em área do Sistema Aquífero Bauru (SAB)
}

\section{Conceptual hydrological model of Santa Barbara Ecological Station (EEcSB) in Bauru Aquifer System (BAS)}

\author{
Lucas Vituri Santarosa $^{1}$; Rodrigo Lilla Manzione ${ }^{2} \bowtie$ \\ 1 Universidade Estadual Paulista (UNESP), Faculdade de Ciências Agronômicas (FCA), Campus de Botucatu, SP, Brasil. \\ 2 Universidade Estadual Paulista (UNESP), Campus de Ourinhos, SP, Brasil.
}

$\triangle$ lucasviturisantarosa@gmail.com, rlmanzione@gmail.com

Resumo

Palavras-chave:

Modelo hidrogeológico conceitual.

Sistema Aquífero Bauru.

Modelagem das águas subterrâneas.

\begin{abstract}
A modelagem hidrogeológica possibilita responder várias questões capazes de descrever a variabilidade espacial e temporal do comportamento dos aquíferos, como as variações nos níveis, a direção e velocidade dos fluxos, a origem da água e volume disponível. A gestão das águas subterrâneas pode ser beneficiada pelas informações geradas pela construção de um Modelo Hidrogeológico Conceitual (MHC), buscando maximizar os dados e experiências, utilizando ambos de forma sistemática para obtenção de respostas e conhecimentos sobre a dinâmica das águas subterrâneas. Este estudo teve como objetivo construir um MHC utilizando técnicas de análise espacial aplicadas a variáveis ambientais e dados de nível freático. Buscou-se com a utilização de uma metodologia quantitativa e qualitativa gerar um MHC para descrever a dinâmica do Sistema Aquífero Bauru (SAB) na Estação Ecológica de Santa Bárbara (EEcSB), município de Águas de Santa Barbara/SP. Os resultados forneceram um MHC que permite uma serie de interpretações iniciais sobre o comportamento do escoamento subterrâneo, subsidiando a tomada de decisões para proteção dos recursos hídricos nos limites da EEcSB. Conclui-se que as descargas por fluxos subterrâneos recentes abastecem as drenagens superficiais presentes na área e este fluxo subterrâneo acompanha a topografia em direção às drenagens principais.
\end{abstract}

Abstract

Keywords:

Hydrogeological Conceptual model.

Bauru Aquifer System.

Groundwater modeling.

Revisado por pares.

Recebido em: 20/02/2017

Aprovado em: 26/11/2017.

\begin{abstract}
Hydrogeological modeling enable to answer several questions capable to describe the spatial and temporal variability of aquifer behavior, such as variations in levels, direction and velocity of flows, water source and available volume. Groundwater management can be benefit by the information generated from the construction of a Hydrogeological Conceptual Model (HCM), looking for data and expert knowledge maximization, using both in a systematic way to obtain answers and knowledge of groundwater dynamics. This study aimed to construct an HCM using spatial analysis techniques applied to environmental variables and water table depths data. From the use of a quantitative and qualitative methodology we search for generating a HCM to describe the Bauru Aquifer System (BAS) dynamics at Santa Bárbara Ecological Station (EEcSB), municipality of Aguas de Santa Barbara/SP, Brazil. The results provide a HCM that allows a series of initial interpretations on the behavior of the underground flow, subsidizing the decision making for the protection of water resources within the boundaries of the EEcSB. It is concluded that discharges through recent underground flows supply the surface drainage present in the area and this underground flow follow the topography towards the major drainages.
\end{abstract}

DOI: http://dx.doi.org/10.14295/ras.v31i4.28826

\section{INTRODUÇÃO}

A importância vital dos recursos hídricos impõe a necessidade crescente de elaboração e aplicação de modelos capazes de descrever suas dinâmicas e fornecer dados fundamentais para sua gestão. Fato que se acentua quando está em pauta os recursos hídricos em ambientes subterrâneos, considerados estoques estratégicos para atender a demanda imposta pela sociedade e manter funções ecológicas.

Diante da complexidade de fatores naturais e antropogênicos que governam e interferem na movimentação e nos estoques das águas subterrâneas, existe a necessidade de levantamento de dados que permitam melhorar a qualidade e quantidade de informações disponíveis. A modelagem hidrogeológica possibilita responder várias questões capazes de descrever a variabilidade espacial do sistema de fluxos, por exemplo, como os níveis da água nos poços se comportam no tempo e espaço em situação de estresse hídrico e a evolução dos níveis freáticos ao longo do tempo. Para isso, é importante o desenvolvimento de modelos hidrogeológicos capazes de reunir informações que descrevam o comportamento dos aquíferos, como as variações nos níveis, a direção e velocidade dos fluxos, a origem da água e volume de água disponível. (ANDERSON; WOESSNER, 1992; KRESIC; MI- 
KSZEWSKI, 2013).

A gestão das águas subterrâneas pode ser beneficiada pela construção de um Modelo Hidrogeológico Conceitual (MHC), que são modelos formados pela reunião de um conjunto de dados espaciais que permitem a interpretação das dinâmicas dos aquíferos. O MHC contém diversas interpretações e provas de sua validade qualitativa e subjetiva alcançada por meio da aplicação de técnicas de investigação específicas, utilizadas para a construção de um modelo numérico que deve ser validado comparando os resultados obtidos pela modelagem em relação às observações em campo (BREDEHOEFT, 2005; VARGAS et al., 2012).

Não só dados, mas também experiências, podem ser usados de forma sistemática para obtenção de respostas que maximizem os benefícios públicos e conhecimentos das dinâmicas das águas subterrâneas (LEGRAND; ROSEN, 2000). As construções de modelos hidrogeológicos buscam atender a essa proposta, como nos estudos realizados para os três dos aquíferos mais importantes para a região oeste do estado de São Paulo, realizados por: Fernandes et al. (2011) e Lastoria et al. (2006) sobre o aquífero Serra Geral; Gastmans et al. (2012) sobre o Sistema Aquífero Guarani (SAG); e Silva (2003), Stradioto (2007) e Carvalho (2014) sobre o Sistema Aquífero Bauru (SAB).

O presente estudo teve como objetivo construir um MHC utilizando técnicas de análise espacial aplicadas a variáveis ambientais e ao nível freático, visando combinar metodologias quantitativas e qualitativas capazes de gerar um MHC para descrever a dinâmica do Sistema Aquífero Bauru (SAB) na Estação Ecológica de Santa Bárbara (EEcSB), município de Águas de Santa Barbara/SP, e, consequentemente, gerar informações para o entendimento do comportamento das águas subterrâneas e subsidiar a gestão dos recursos hídricos na unidade de conservação ambiental.

\section{CARACTERIZAÇÃO DA ÁREA DE ESTUDO}

A Estação Ecológica de Santa Bárbara (EEcSB) está localizada à Rodovia SP $261-\mathrm{km} \mathrm{58}$, latitude $24^{\circ} 48^{\prime} \mathrm{S}$ e longitude $49^{\circ} 13^{-}$ O, no município de Águas de Santa Bárbara - São Paulo - Brasil. A EEcSB possui uma área de 2.712 hectares de vegetação nativa (cerrado, brejos e mata de galeria) dividindo o espaço com o re- florestamento com espécies exóticas (pinus e eucalipto), totalizando 4.371 hectares destinados para a Floresta Estadual e Estação Ecológica instaladas no munícipio.

Toda a área faz parte da unidade hidrográfica do rio Pardo (ao sul da EEcSB), na Unidade de Gerenciamento de Recursos Hídricos do Médio Paranapanema (UGRHI-17). Os maiores corpos de água em contato com a Estação Ecológica são o rio Capivari e o rio Capão Rico (HONDA; NIZOLI, 2005). Ambos os rios nascem fora da Unidade de Conservação e a margeiam, sendo que o rio Capivari é o limite a oeste e o rio Capão Rico, a leste.

A EEcSB localiza-se sobre o Sistema Aquífero Bauru (SAB), um sistema aquífero sedimentar de extensão regional que ocupa a maior parte da região oeste do território do Estado de São Paulo, com uma área aproximada de 96 mil km² (IRITANI; EZAKI, 2008). O SAB representa uma das reservas hídricas mais importantes do Estado, servindo como fonte de abastecimento público para muitos municípios. Na região do Médio Paranapanema (UGRHI17) o SAB é utilizado para suprir $75 \%$ da sua demanda socioeconômica (FIESP, 2005).

De acordo com Silva et al. (2005), o SAB é formado pelos aquíferos Marília, Adamantina, Birigui, Santo Anastácio e Caiuá, e os aquitardos Araçatuba e Pirapozinho. As características gerais do SAB apresentam porosidade efetiva de $5 \%$ a $15 \%$; transmissividade de 10 a $300 \mathrm{~m}^{2} /$ dia; permeabilidade de 0,1 a $3 \mathrm{~m} /$ dia; capacidade especifica entre $0,02 \mathrm{~m}^{3} / \mathrm{h} / \mathrm{m}$ a $4,9 \mathrm{~m}^{3} / \mathrm{h} / \mathrm{m}$; condutividade hidráulica de 0,02 a 3,66 m/dia e; vazão média explorável em torno de $80 \mathrm{~m}^{3} / \mathrm{h}$ (DAEE, 2005).

Na região da EEcSB, o SAB é composto por arenitos das Formações Adamantina (Vale do Rio do Peixe) e Marilia pertencentes ao Grupo Bauru (MELO; DURIGAN, 2011; CPRM, 2006; IPT, 1981). Existe ainda a ocorrência da Formação Serra Geral de origem ígnea, extrusiva, formada por derrames de lava basáltica ( $\mathrm{Fi}$ gura 1).

0 aquífero Adamantina é predominante na área das bacias estudadas, considerado livre a semiconfinado e continuo apresentando um comportamento hidráulico bastante heterogêneo e anisotrópico, mesmo em localidades relativamente próximas, devido a variações das concentrações de argila nos arenitos (SILVA, 2003). 
Figura 1 - Localização regional da EEcSB

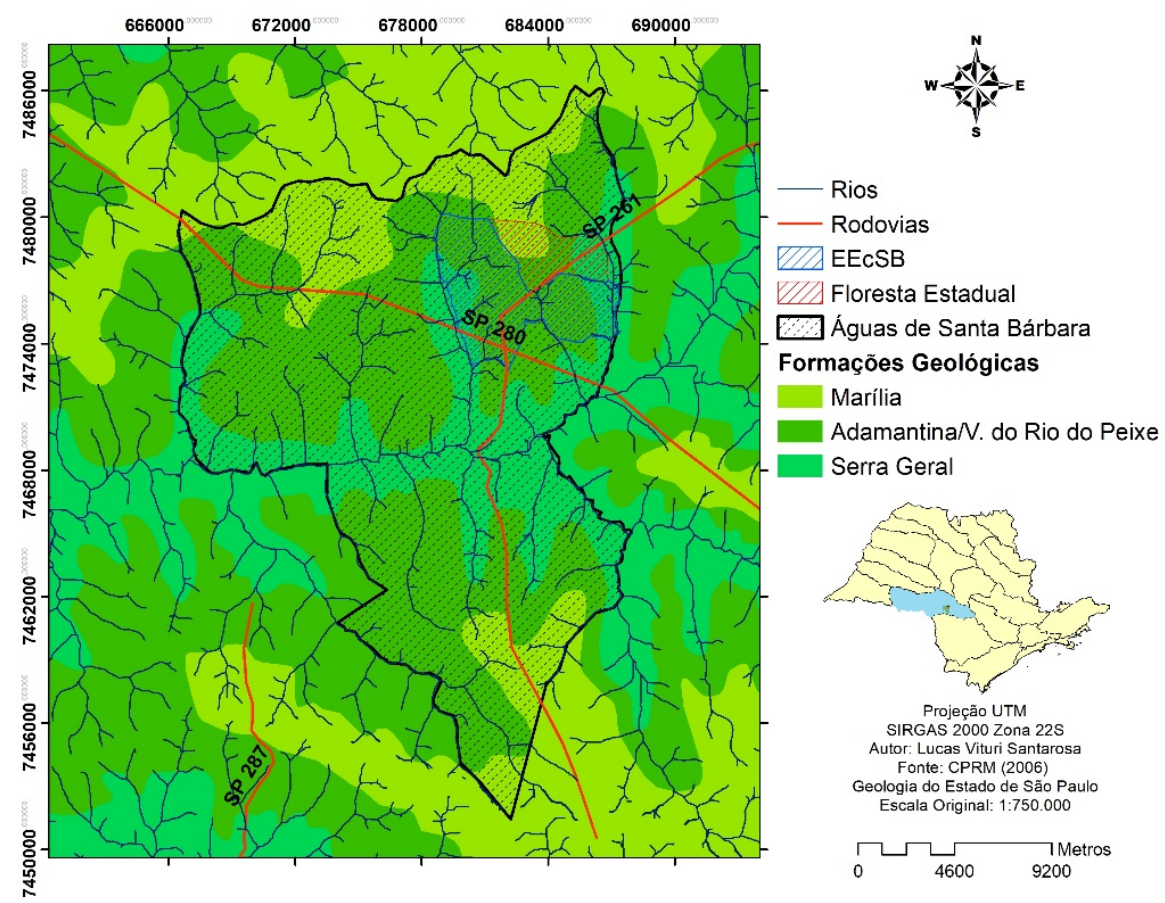

\section{FUNDAMENTAÇÃO TEÓRICA}

Para elaborar MHC são necessárias previsões realizadas a partir da organização de dados geológicos e hidrológicos com a capacidade de interpretar as inter-relações dos principais fatores, bem como a associação e padrões destes fatores (LEGRAND; ROSEN, 2000). As águas subterrâneas estão sujeitas às grandezas físicas que governam o movimento dos fluidos, determinando o fluxo subterrâneo, a infiltração, transporte de contaminantes, etc. (MANZIONE, 2015). Para realizar a construção do MHC apresentado neste estudo, foram reunidas algumas propriedades capazes de descrever a dinâmica da água subterrânea na área de estudo: carga hidráulica, espessura da camada saturada, transmissividade e porosidade especifica do aquífero.

A porosidade é uma das propriedades fundamentais do meio saturado. Definida como espaço intergranular ou vazios do solo ou das rochas (FETTER, 2001; TODD; MAYS, 2005), a porosidade do aquífero determina o montante de água armazenada e sua velocidade de movimentação, influenciando diretamente na condutividade hidráulica e, consequentemente, nas características de permeabilidade e transmissividade. 0 diâmetro dos sedimentos condiciona o diâmetro dos poros. Deste modo, a permeabilidade é mensurada pela relação entre o diâmetro médio das partículas e a disposição do material (FREEZE; CHERRY, 1979). Característica diretamente relacionada com a produção e armazenamento específico de um aquífero, limitando o montante a ser explorado ou as taxas de rebaixamento (FETTER, 2001; TODD; MAYS, 2005).

Segundo a Lei de Darcy, a vazão em um meio poroso é calculada a partir da condutividade hidráulica, a área da seção transversal e o gradiente hidráulico, obtido pela diferença das cargas hidráulicas dividida pelo comprimento da seção (FETTER, 2001; CABRAL, 2000).
A condutividade hidráulica representa a capacidade de um meio poroso em transmitir água através dos espaços vazios (SALARASHAYERI; SIOSEMARDE, 2012). A condutividade hidráulica associada a espessura saturada de um aquífero determina sua capacidade de transmissividade.

A carga hidráulica é definida por Cabral (2000) como uma energia mecânica composta pelas energias cinética, potencial gravitacional e de pressão. Ela determina a direção do fluxo da água subterrânea, o movimento segue o gradiente hidráulico de áreas com maior carga hidráulica para áreas com menor carga hidráulica, esta diferença é chamada de gradiente hidráulico. Em aquíferos livres a carga hidráulica do nível freático é igual a altitude do nível da água (FREEZE; CHERRY, 1979; SNYDER, 2008), portanto, o relevo pode ser um fator determinante na movimentação das águas subterrâneas.

As medições da carga hidráulica podem ser realizadas com a utilização de piezômetros. A elevação do nível freático pode ser usada para inferir a direção da água possibilitando diversas interpretações para a tomada de decisões. Os valores do nível freático (ou potenciométrico) formam linhas equipotenciais, os fluxos movimentam-se perpendicularmente a estas linhas formando redes de fluxo, especialmente em meios isotrópicos. Através destas redes é possível identificar zonas de recarga, de trânsito e de descarga, bem como os divisores de água subterrânea; determinar a velocidade aparente do fluxo; e selecionar locais mais adequados para extração de água ou regiões com suscetibilidade à poluição (MANZIONE, 2015).

Com base nestas propriedades foi possível produzir diferentes informações para elaboração do MHC conforme a metodologia adotada. 


\section{METODOLOGIA}

Para a elaboração do MHC foi utilizado o pacote Groundwater do SIG ArcGIS 10.3. O pacote apresenta quatro ferramentas: (1) Darcy Flow calcula o volume residual para o regime de fluxo constante; (2) Darcy Velocity calcula o vetor de velocidade para cada célula segundo a Lei de Darcy; (3) Particle Tracking simula a trajetória de uma partícula baseado nos dados gerados pela ferramenta Darcy Velocity e; (4) Porous Puff que faz a simulação da dispersão bidimensional de um poluente no meio poroso (GIMENES et al, 2012; ESRI, 2016).

Foram utilizadas para estimar a direção dos fluxos subterrâneos as ferramentas Darcy Velocity e Particle Tracking. A ferramenta Darcy Velocity necessita da carga hidráulica, da porosidade efetiva, espessura saturada e transmissividade para calcular a magnitude e direção do fluxo subterrâneo, esta ferramenta foi aplicada para compreender como as características locais influenciam na dinâmica da água subterrânea simulando vetores de direção do fluxo. Com os resultados gerados, foram feitas simulações de escoamento para entender o comportamento do fluxo utilizando a ferramenta Particle Tracking para verificar a trajetória preferencial da água subterrânea.

É importante salientar que o pacote Groundwater tem uma abordagem bidimensional baseada em dados espaciais. A análise mostra-se limitada em relação ao componente vertical do fluxo, prejudicando os resultados do MHC para realização de análise em três dimensões. Outra característica importante do pacote é que ele considera o meio isotrópico, característica que foi considerada para a formulação do MHC baseando-se na variação muito sutil nas características do solo ao longo da área de estudo e da predominância da Formação Adamantina.

Para complementar esta análise, a partir de uma abstração dos dados reunidos, foram elaborados perfis longitudinais inferindo empiricamente o comportamento dos fluxos subterrâneos.

\subsection{Base de dados}

Para a construção do MHC foram utilizados dados referentes a profundidade do nível freático, a espessura da camada saturada, valores de porosidade efetiva e de condutividade hidráulica, além de um Modelo Digital do Terreno (MDT).

Estes dados foram relacionados para gerar os valores da carga hidráulica, estimada pela diferença entre a altitude do terreno e a profundidade do nível freático, considerando que para aquíferos livres a carga hidráulica é igual ao nível potenciométrico; a espessura saturada, estimada pela definição da espessura média da camada de arenito sobreposta a camada confinante de basalto da Formação Serra Geral; a transmissividade, obtida pela multiplicação dos valores de condutividade hidráulica pela espessura da camada saturada; e a porosidade específica do aquífero deduzida a partir da literatura consultada.

Esses dados fazem parte do levantamento, generalizações e resultados apresentados em Santarosa (2016), que realizou o teste de diferentes variáveis físico-hídricas do solo e do relevo na interpolação do nível freático por meio da aplicação de diferentes interpoladores geoestatísticos. Neste é possivel verificar os métodos de interpolação (krigagem) utilizados para gerar os diferentes dados utilizados no pacote Groundwater.

A profundidade do nível freático foi medida em 48 poços de monitoramento com piezômetros instalados nas áreas das bacias do Guarantã, Santana, Boi, Passarinho e Bugre (Figura 2) instalados na camada superficial na interface do solo para Formação Adamantina com profundidade mínima de 4 e máxima de 8 metros.

Para este estudo foi utilizada a medição do nível freático iniciada em 09 de setembro de 2014 até o dia 03 de dezembro de 2015. o Gráfico 1 apresenta a variação do nível freático, calculado a partir da média do nível dos 48 poços.

Gráfico 1 - Oscilação do nível freático e precipitação no período de 09 de setembro de 2014 a 03 de dezembro de 2015

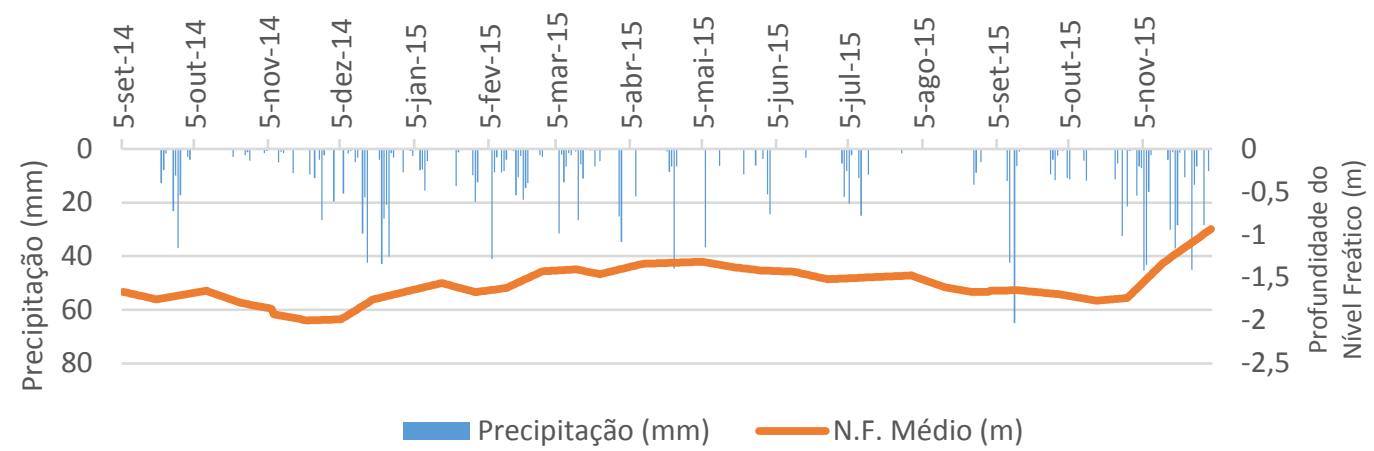


Figura 2 - Localização dos poços de monitoramento e pontos de coleta de dados na EEcSB

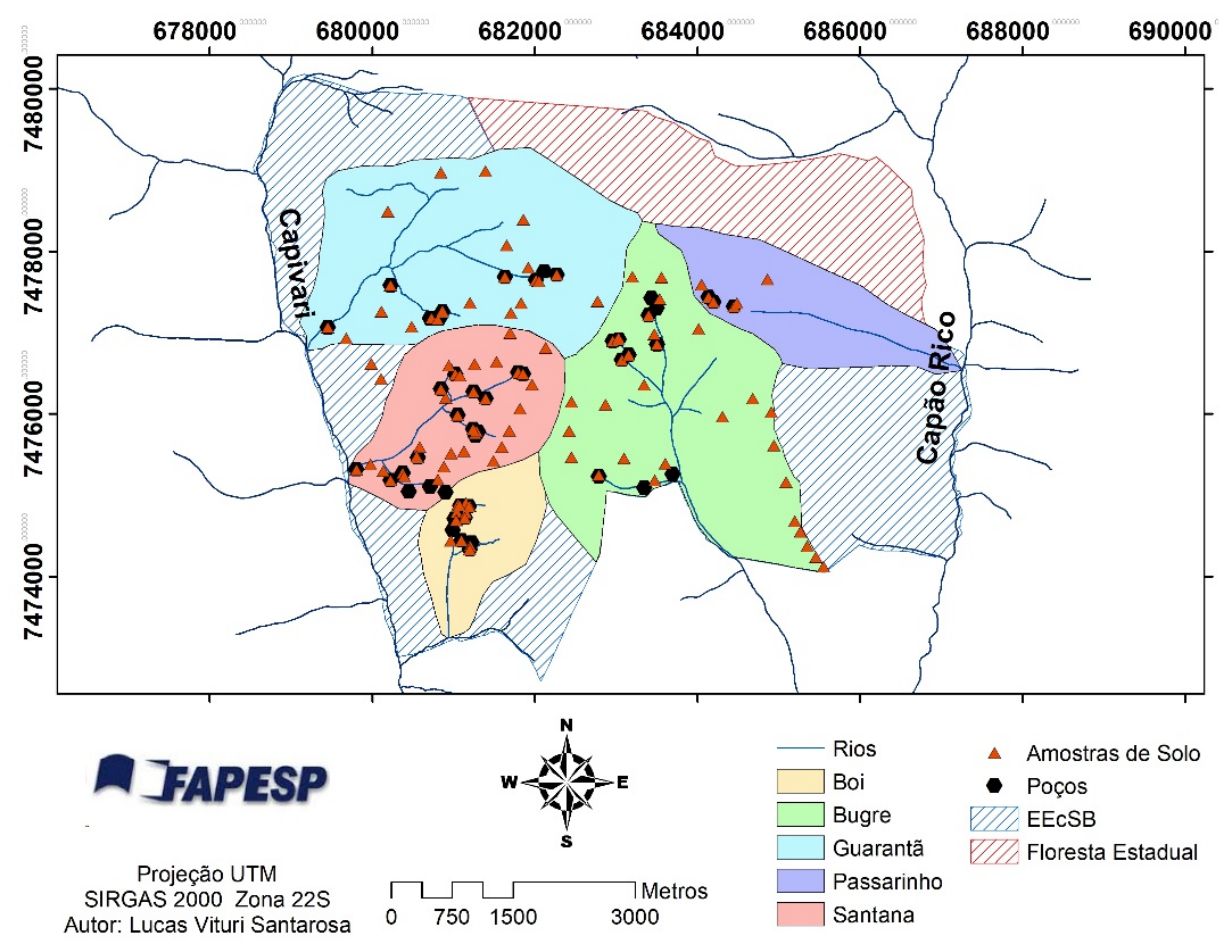

A espessura da camada saturada, determinada pela espessura da camada do arenito da Formação Adamantina (Vale do Rio do Peixe) (Figura 3), foi estimada a partir dos dados dos perfis de poços do Sistema de Informação de Águas Subterrâneas (SIAGAS) do Serviço Geológico do Brasil (CPRM) presentes na região da EEcSB. A espessura foi determinada pela profundidade da camada do arenito da Formação Adamantina em cada um dos poços disponíveis, foram consultados ao todo 8 poços presente a cabeceira das bacias dos rios Capivari e Capão Rico.

Figura 3 - Espessura da camada do arenito da Formação Adamantina na EEcSB

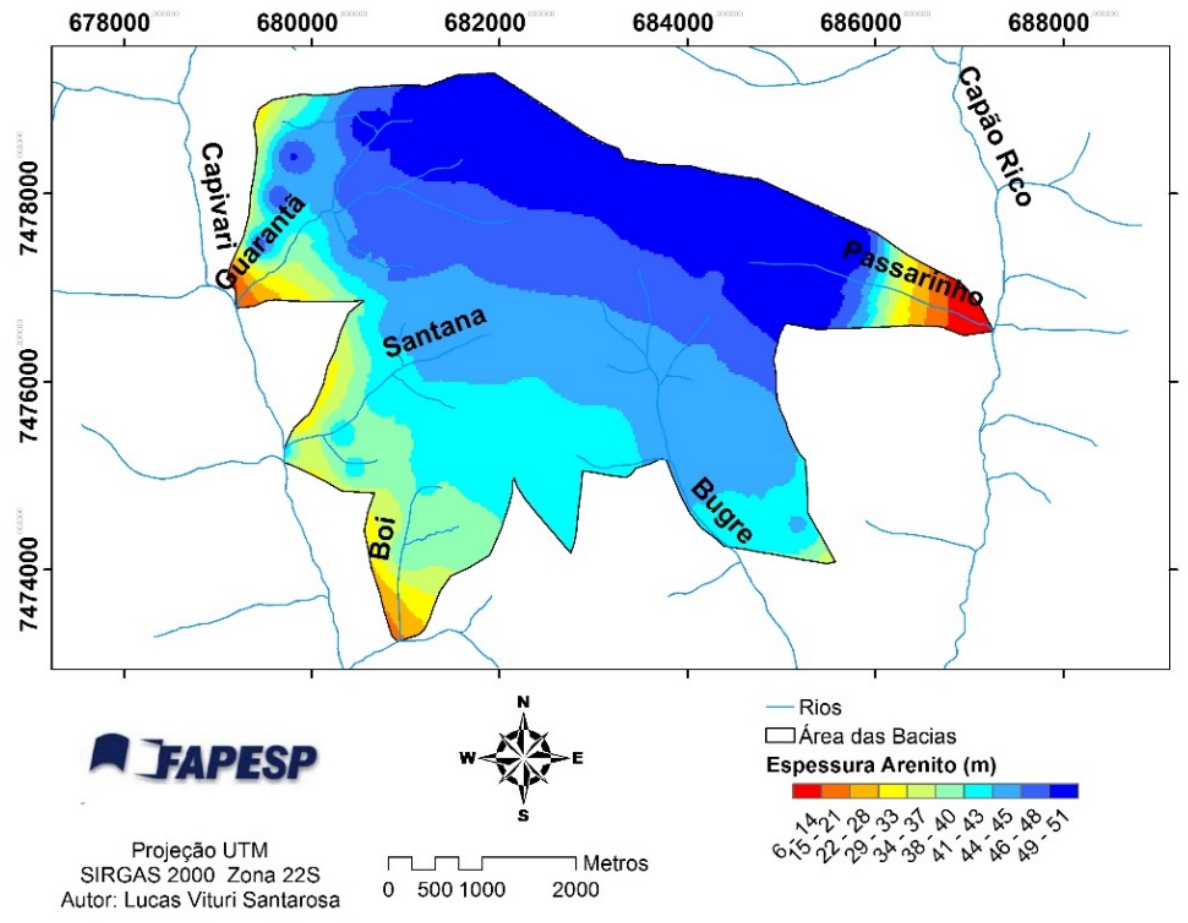

O valor de porosidade efetiva utilizado foi de $10 \%$, que representa um valor médio da porosidade efetiva do SAB, entre $5 \%$ e 15\% (DAEE, 2005), que se aproxima do limite inferior da porosidade efetiva para solos Quartzarênicos, entre 12 e 18\% (MANZIONE et al., 2007), considerada devido a predominância da fração de areia encontrada na análise granulométrica. Esse valor adotado levou em conta a generalização dos dados em relação a camada de solo e de rocha, deste modo o valor da porosidade efetiva não pode ser tão baixo para simular apenas a condição da rocha e nem tão elevado que simule apenas a condição de poro- 
sidade efetiva do solo.

Para o cálculo da condutividade hidráulica foram utilizadas amostras de solo coletadas nas proximidades dos poços e distribuídas pelas bacias (Figura 2), totalizado 113 amostras coletadas a cerca de 100 centímetros de profundidade. Estas passaram por análise granulométrica segundo a metodologia da Embrapa (1997). Os resultados desta analise foram utilizados para determinar a condutividade hidráulica adaptando o método de Hazen (FETTER, 2001) para a variação granulométrica do solo. 0 método consiste em estimar a condutividade hidráulica a partir do diâmetro efetivo das partículas do solo.

Os valores de condutividade hidráulica calculada apresentam variação de 4 a 5,5 m/dia, enquanto que os valores médios obtidos por Silva (2003) para o Sistema Aquífero Bauru (SAB) apresenta variação de 1 a $4 \mathrm{~m} /$ dia para camadas superiores e de 1 a 3 $\mathrm{m} /$ dia para camadas inferiores.

O Modelo Digital do Terreno (MDT) foi elaborado a partir das Car tas Topográficas do Instituto Brasileiro de Geografia e Estatística (IBGE) com escala de 1:50.000.

Os dados utilizados para aplicação da metodologia são generalizações, adaptações de metodologias e dados disponíveis. No presente estudo a coleta de dados foi limitada pelos recursos disponíveis, portanto, a intenção deste primeiro levantamento consiste em gerar informações iniciais a partir de uma exploração fundamentada teoricamente e associada aos dados disponí veis e coletados em campo correlacionadas a estudos sobre o $\mathrm{SAB}$.

\section{RESULTADOS E DISCUSSÃO}

Com base nas informações reunidas os dados foram processados no pacote Groundwater utilizando as ferramentas Darcy Velocity, que calcula os vetores de direção da água subterrânea baseados na Lei de Darcy (Figuras 4), e Particle Tracking, que estima o caminho de uma partícula baseado nos vetores de direção (Figura 5), estimando a trajetória da água subterrânea.

Figura 4 - Mapa potenciométrico e vetores dos fluxos subterrâneo do SAB na EEcSB para o dia $03 / 12 / 2015$

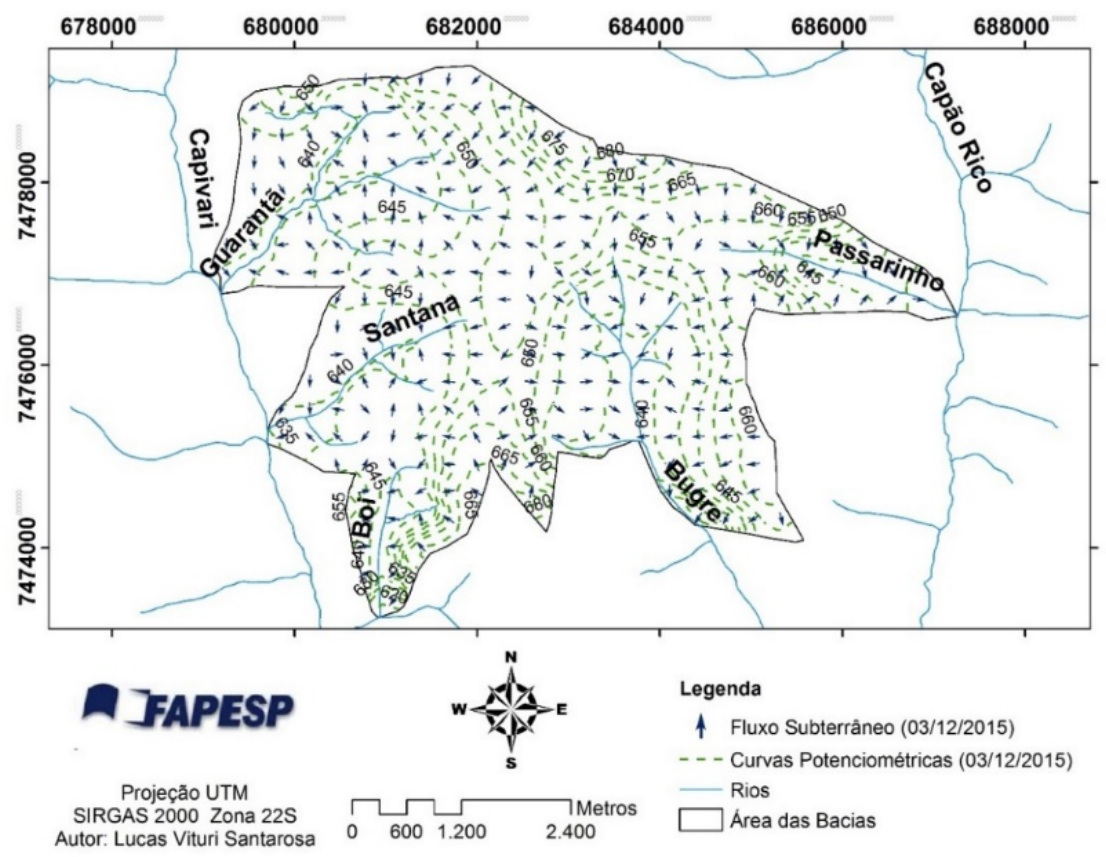

Este comportamento na modelagem do fluxo pode estar associado a relativa homogeneidade dos dados de entrada em relação as características hídricas do material do subsolo, nota-se que o relevo acaba exercendo muita influência sobre o fluxo subterrâneo, apenas em alguns locais o fluxo subterrâneo não segue o padrão superficial.

A qualidade das informações utilizadas para realização da estimativa de fluxo abre precedentes para um levantamento mais detalhado e execução de um modelo mais completo, capaz de fazer a representação mais detalhada dos fluxos. Sobre esta questão Von Asmuth e Knotters (2004) relatam que a coleta e análise de dados para mapeamento e modelagem empírica é um procedimento minucioso, pois o volume de informações contidas em uma descrição completa da dinâmica das águas subterrâneas é muito complexo para uma representação visual, exigindo cuidados para não perder informação.

Analisando os fluxos representados foi possível observar três pa drões:

I. Escoamento na direção das bacias da região oeste, seguindo o sentido das bacias do Guarantã, Santana e Boi em direção do rio Capivari;

II. Escoamento na direção da Bacia do Bugre com maior recepção dos fluxos gerados ao norte da área indo no sentido sul; e

III. Uma representatividade menor de fluxos no extremo leste, na direção da Bacia do Passarinho, revelando menor volume de água desta região que contribui para vazão 
do rio Capão Rico. Porém, na bacia do Passarinho os dados são escassos aumentando a incerteza na determinação das curvas potenciométricas nesta área.

Com a análise do fluxo nas bacias do Guarantã, Santana e Boi, nota-se a divisão dos sentidos dos fluxos marcada pelo divisor de águas subterrâneas entre essas bacias e a bacia do Bugre. 0 rio Capivari controla o nivel de base dos rios Guarantã, Santana e Boi e influencia o fluxo da água subterrânea. Como o Aquífero Bauru diminui de espessura em direção ao rio Capivari e o Aquífero Serra Geral atua como um obstáculo ao fluxo vertical ocorre predominantemente a descarga nas drenagens.

A Bacia do Bugre na região central da área recebe a maior parte dos fluxos subterrâneos que convergem no sentido da drenagem, nota-se que o divisor de água subterrâneo nesta área não coincide com o superficial, boa parte dos fluxos subterrâneos na área de recarga da bacia do Passarinho convergem para a bacia do Bugre.

Os três perfis longitudinais representam as generalizações sobre a movimentação da água subterrânea nas seções (Figura 6): (AB) da bacia do Guarantã, passando pela Bacia do Santana até a Bacia do Boi; (C-D) da bacia do Guarantã, passando a montante pela bacia do Bugre até a nascente do Ribeirão Passarinho e; (EF) da bacia do Santana até a Bacia do Bugre. Os perfis longitudinais simulam a disposição das camadas de acordo com os dados disponiveis e os fluxos subterrâneos gerados empiricamente pela interpretação dos resultados obtidos (Figuras 7, 8 e 9).

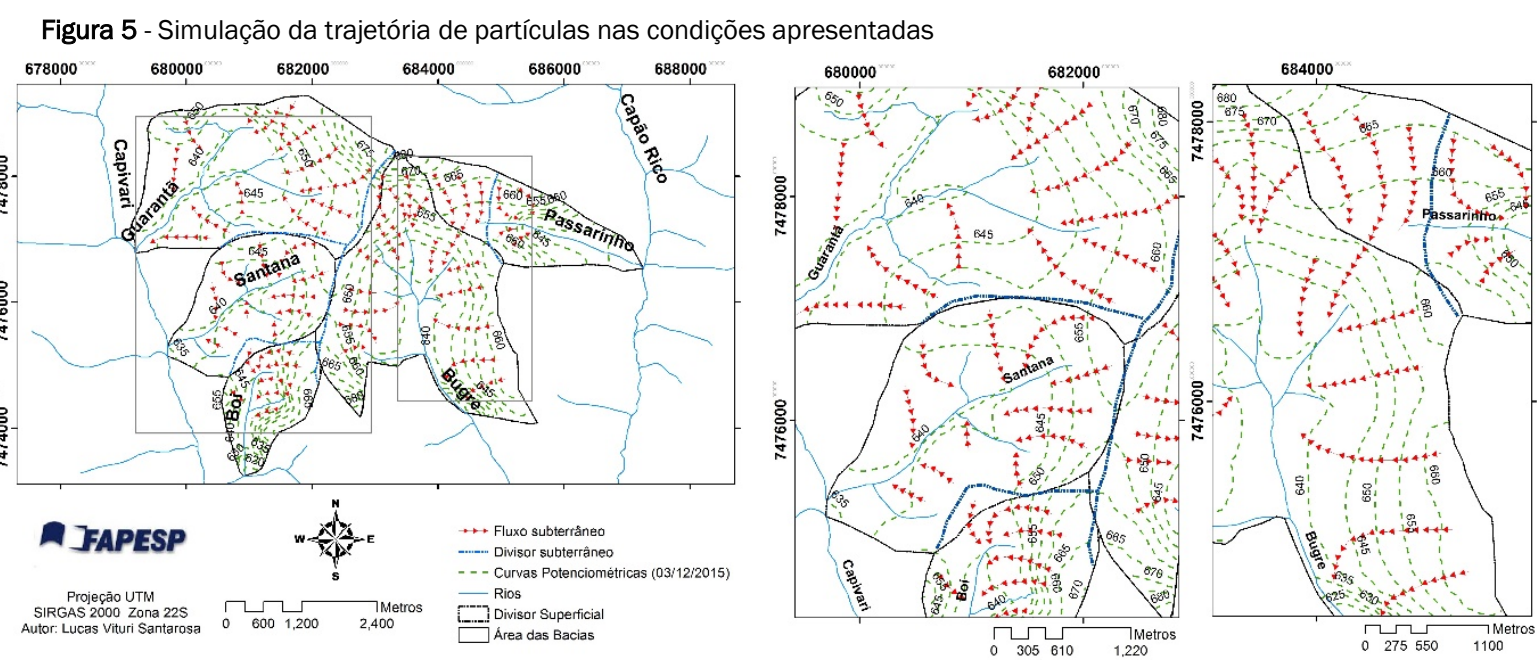

Figura 6 - Secções escolhidas para elaboração dos perfis longitudinais

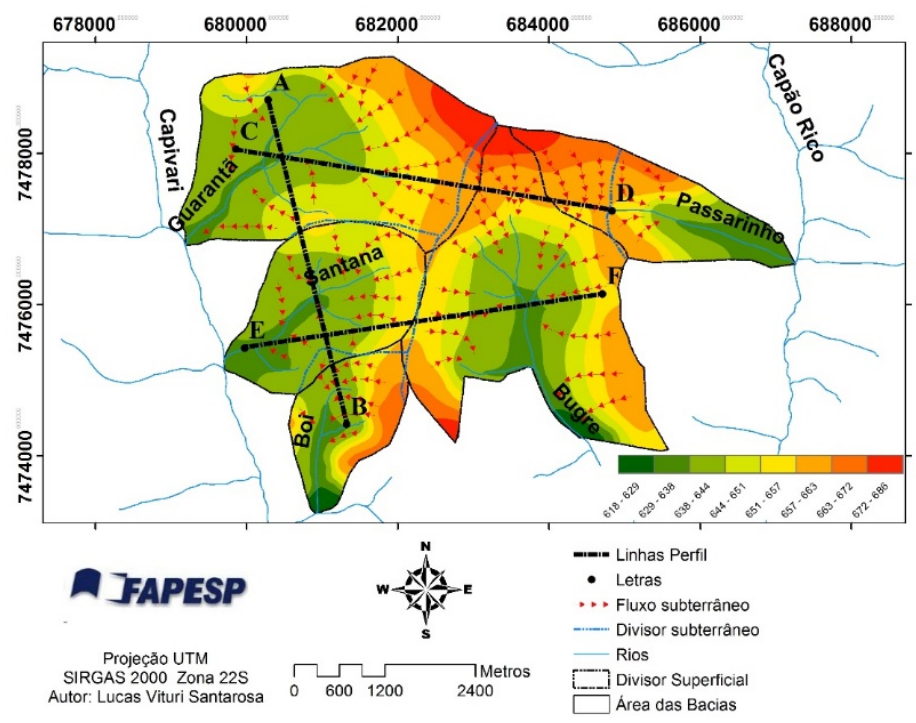

No perfil A-B (Figura 7), mostra os fluxos na direção das drenagens e acredita-se na existência de fluxos de drenagem profunda na formação Serra Geral a partir de fraturas conectando o aquífero Bauru ao Aquífero Serra Geral. Esta afirmação está pautada nos níveis freáticos encontrados na bacia do Santana conforme apresentado por Santarosa (2016), este comportamento foi considerado nos demais perfis.
O perfil C-D (Figura 8) representa o comportamento do fluxo entre a bacia do Guarantã, passando pela montante da bacia do Bugre

até a nascente do Ribeirão Passarinho. Baseado no gradiente hidráulico proporcionado pelo relevo, é possível inferir que os fluxos preferenciais acompanham o relevo no sentido do Ribeirão Guarantã. E no perfil E-F (Figura 9) a predominância de fluxos no sentido da drenagem do Ribeirão Bugre. 
Figura 7 - Perfil I ongitudinal A-B

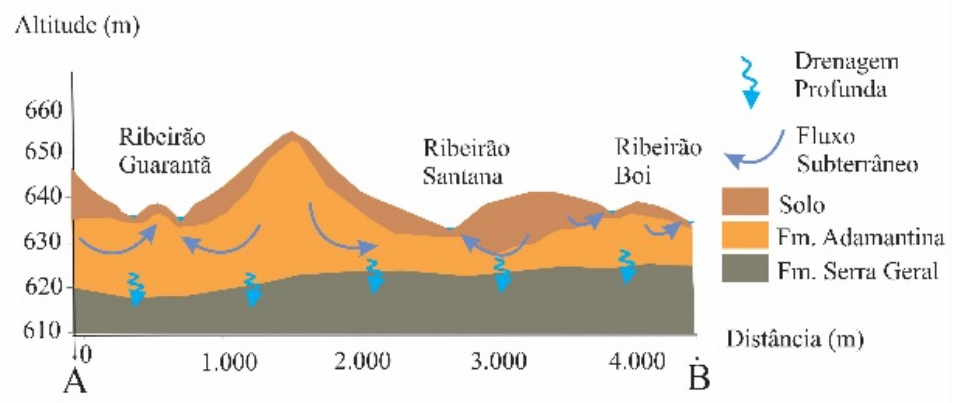

Figura 8 - Perfil longitudinal C-D

Figura 9 - Perfil longitudinal E-F

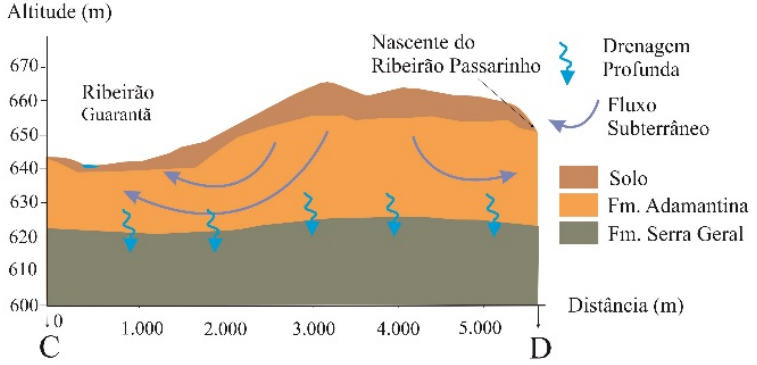

De modo geral, baseando-se na oscilação do nível freático, informações do mapa potenciométrico, da trajetória dos fluxos e dos perfis A-B, C-D e E-D, infere-se que ocorram descargas de fluxos subterrâneos recentes (semanas a meses) que abastecem as drenagens superficiais e que o fluxo subterrâneo preferencial está organizado na direção das drenagens principais acompanhando a topografia, comportamentos típicos de aquíferos livres.

Este levantamento pode ser considerado uma importante fonte de informações e com qualidade física suficiente para interpretação de algumas das dinâmicas do SAB na EEcSB. O caráter pioneiro aliado a baixa captação de recursos possibilita apenas uma interpretação inicial sobre o comportamento do escoamento subterrâneo, um subsídio fundamental para tomada de decisões voltadas para a proteção dos recursos hídricos nos limites da EEcSB. Por utilizar dados generalizados e com baixa resolução espacial, os dados servem para inferências primárias abrindo precedentes para aprofundamento do levantamento de dados mais precisos para uma modelagem mais completa.

O estudo apresenta uma metodologia que reúne dados de forma sistemática para obtenção de respostas que tragam benefícios públicos a partir do conhecimento da dinâmica das águas subterrâneas. Informações capazes de responder várias questões fundamentais para a gestão das águas subterrâneas, como identificar áreas de recarga e descarga e a movimentação de contaminantes presentes na água oriundos de atividades realizadas na borda EEcSB, capaz de proteger os recursos hídricos locais e fazer vislumbrar essas áreas como produtoras e protetoras dos recursos hídricos.

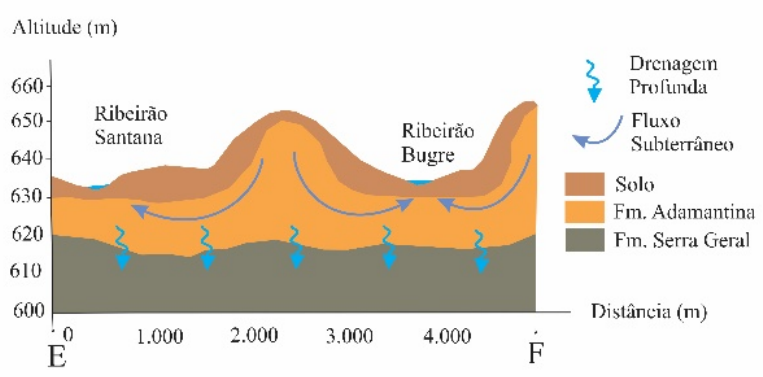

\section{CONCLUSÕES}

Este estudo apresenta um conjunto de dados gerados a partir de levantamentos realizados por meio de métodos diretos e informações disponíveis para gerar um Modelo Hidrogeológico Conceitual (MHC) do Sistema Aquífero Bauru (SAB) na Estação Ecológica de Santa Barbara (EEcSB).

$\mathrm{Na}$ área da EEcSB as descargas de fluxos subterrâneos recentes abastecem as drenagens superficiais e o fluxo subterrâneo acompanha a topografia em direção às drenagens principais, as bacias do Guarantã, Santana e Boi ocorrem em direção ao rio Capivari, enquanto as bacias do Bugre e do Passarinho contribuem com a bacia do rio Capão Rico. Identificou-se características de um aquífero livre e raso com grande influência das características superficiais em suas dinâmicas, com sensibilidade as variações climáticas e ações antrópicas.

\section{AGRADECIMENTOS}

Agradecemos ao grupo de estudos $\mathrm{LABH} 2 \mathrm{O}$ e os envolvidos na coleta de dados em campo, ao Instituto Florestal do Estado de São Paulo (IF) pelo acesso a área de estudo e cooperação científica, e a Fundação de Amparo à Pesquisa do Estado de São Paulo (FAPESP), pelo suporte financeiro (processo 2014/04524-7) e pela bolsa de mestrado (processo 2015/05171-3) concedidos para execução dessa pesquisa.

\section{REFERÊNCIAS}

ANDERSON, M. P.; WOESSNER, W. W. Applied groundwater modeling: simulation of flow and advective transport. San Diego: Academic Press, 1992. 
BREDEHOEFT, J. The conceptualization model problem surprise. Hydrogeology Journal, v. 13, n. 1, p. 37-46, mar. 2005.

CABRAL, J. Movimento das águas subterrâneas. In: FEITOSA, F. A. C. Hidrogeologia: conceitos e aplicações. 2 ed. Rio de Janeiro: CPRM, 2000. p.34-51.

CARVALHO, A. M. de. Modelagem numérica como ferramenta para a gestão das águas subterrâneas em São José do Rio Preto - SP. 2014. Dissertação (Mestrado em Hidrogeologia e Meio Ambiente) - Instituto de Geociências, Universidade de São Paulo, São Paulo, 2014. doi: 10.11606/D.44.2014.tde-25092014-101823. Acesso em: 2017-0106.

CPRM (Serviço Geológico do Brasil) Domínio geológico litoestratográfico do Estado de São Paulo. São Paulo, 2006. Escala 1:750.000.

DAEE (Departamento de Águas e Energia Elétrica); IG (Instituto Geológico); IPT (Instituto de Pesquisas Tecnológicas); CPRM (Serviço Geológico do Brasil). Mapa de águas subterrâneas do Estado de São Paulo escala: 1:1.000.000: nota explicativa. São Paulo: Conselho Estadual de Recursos Hídricos, 2005.

EMBRAPA (EMPRESA BRASILEIRA DE PESQUISA AGROPECUÁRIA). Manual demétodos de análises de solo. 2.ed.. Rio de Janeiro: Ministério da Agricultura e do Abastecimento, 1997. 212 p.

ESRI (ENVIRONMENTAL SYSTEMS RESOURCE INSTITUTE). An overview of the Groundwater toolset. Disponivel em: <http://desktop.arcgis.com/en/arcmap/10.3/tools/spatial-analyst-toolbox/an-overview-of-the-groundwater-tools.htm>. Acesso em: 3 jun. 2016.

FERNANDES, A. J.; MALDANER, C. H.; ROULEAU, A. Análise das fraturas nos basaltos de Ribeirão Preto, SP: aplicação à elaboração de modelo hidrogeológico conceitual. Geologia USP. Série Científica, v. 11, n. 3, p. 43-64, 1 dez. 2011.

FETTER, C. W. Applied hydrogeology. 4th ed ed. Upper Saddle River, N.J: Prentice Hall, 2001.

FIESP- Federação das Indústrias do Estado de São Paulo. Orientações para a utilização de Águas subterrâneas no Estado de São Paulo. 2005. 40p.

FREEZE, R. A.; CHERRY, J. A. Groundwater. Englewood Cliffs, N.J: PrenticeHall, 1979.

GASTMANS, D.; VEROSLAVSKY, G.; KIANG CHANG, H.; CAETANO-CHANG, M.R.; NOGUEIRA PRESSINOTTI, M.M. Modelo hidrogeológico conceptual del Sistema Acuífero Guaraní (SAG): una herramienta para la gestión. Boletín Geológico y Minero, v. 123, n. 3, p. 249-265, 2012.

GIMENES, F. B. Q.; AUGUSTO FILHO, O.; SOARES, W. C. Modelagem dos fluxos de água subterrânea na região metropolitana de João Pessoa (PB) utilizando ferramentas de hidrologia em SIG. Revista Brasileira de Geologia de Engenharia e Ambiental, v. 2, n. 2, p. 9-18, 2012.

HONDA, E. A.; NIZOLI, E. C. Caracterização das águas da Estação Ecológica de Águas De Santa Bárbara. Relatório Técnico. São Paulo: Instituto Florestal, 2005. $41 \mathrm{p}$.

IPT (Instituto de Pesquisas Tecnológicas do Estado de São Paulo). Mapa geológico do Estado de São Paulo. São Paulo, 1981. Escala 1:500.000.

IRITANI, M. A.; EZAKI, S. As águas subterrâneas do Estado de São Paulo. São Paulo: Secretaria do Estado do Meio Ambiente - SMA, 2008. 104p.
KRESIC, N.; MIKSZEWSKI, A. Hydrogeological conceptual site models: data analysis and visualization. Leiden: CRC Press, 2013. 600 p.

LASTORIA, G.; SINELLI, O.; HUNG KIANG, C.; HUTCHEON, I.; PARANHOS FILHO, A. C.; GASTMANS, D. Hidrogeologia da formação serra geral no estado de mato grosso do sul. Águas Subterrâneas, v. 20, n. 1, 8 nov. 2006.

LEGRAND, H. E.; ROSEN, L. Systematic Makings of Early Stage Hydrogeologic Conceptual Models. Ground Water, v. 38, n. 6, p. 887-893, 1 nov. 2000.

MANZIONE, R. L. Águas subterrâneas: conceitos e aplicações sob uma visão multidisciplinar. 1a edição ed. Jundiaí, SP: Paco Editorial, 2015.

MANZIONE, R. L.; DRUCK, S.; CÂMARA, G.; MONTEIRO, A. M. V. Modelagem de incertezas na análise espaço-temporal dos níveis freáticos em uma bacia hidrográfica. Pesquisa Agropecuária Brasileira, v. 42, n. 1, p. 25-34, 2007.

MELO, A. C. G.; DURIGAN, G. Plano de manejo da estação ecológica de Santa Bárbara. São Paulo: Instituto Florestal, 2011. 222p.

SALARASHAYERI, A. F.; SIOSEMARDE, M. Prediction of soil hydraulic conductivity from particle-size distribution. World Acad. Sci. Eng. Technol, v. 61, n. 61, p. 454-458, 2012.

SANTAROSA, L. V. Mapeamento de niveis freáticos do Sistema Aquífero Bauru (SAB) em área de proteção ambiental em Águas de Santa Bárbara/SP durante o ano hidrológico 2014/15. Dissertação de mestradoBotucatu: Universidade Estadual Paulista, Faculdade de Ciências Agronômicas, 2016

SILVA, F. de P. e. Geologia de subsuperfície e hidroestratigrafia do grupo Bauru no estado de São Paulo. 2003. xiv, 167 f. Tese (doutorado) - Universidade Estadual Paulista, Instituto de Geociências e Ciências Exatas, 2003

SILVA, F. de P. e.; HUNG KIANG, C.; CAETANO-CHANG, M. R. Hidroestratigrafia do Grupo Bauru (K) no Estado de São Paulo. Águas Subterrâneas, v. 19, n. 2, 28 jun. 2005.

SNYDER, D.T. Estimated depth to ground water and configuration of the water table in the Portland, Oregon area: U.S. Geological Survey Scientific Investigations Report 2008-5059, 2008, 40 p. Disponível em: <http://pubs.usgs.gov/sir/2008/5059/> Acesso em: 04 de abr. 2016

STRADIOTO, M. R. Hidroquímica e aspectos diagenéticos dos sistema aqüífero Bauru na região sudoeste do estado de São Paulo. 2007. ix, 103 f. Dissertação (mestrado) - Universidade Estadual Paulista, Instituto de Geociências e Ciências Exatas, 2007. Disponivel em: <http://hdl.handle.net/11449/92769>.

TODD, D. K.; MAYS, L. W. Groundwater hydrology. 3rd ed ed. Hoboken, $\mathrm{NJ}$ : Wiley, 2005.

VARGAS, T. B.; MARTINEZ, J.F.E.; TOBON, C.A.P. Conceptual Models in: Hydrogeology, Methodology and Results In: Hydrogeology - A Global Perspective . Croacia: INTECH, 2012. p.203 - 222.

VON ASMUTH, J. R.; KNOTTERS, M. Characterising groundwater dynamics based on a system identification approach. Journal of Hydrology. V.296, p, 34-118, 2004. 